\title{
Real-time Rotation Measurement using Digital Image Correlation
}

\author{
Da Yang ${ }^{1}$, and Dongsheng Zhang ${ }^{1,2 *}$ \\ ${ }^{1}$ Shanghai Institute of Applied Mathematics and Mechanics, \\ School of Mechanics and Engineering Science, Shanghai University, Shanghai, 200444, China \\ ${ }^{2}$ Shanghai Key Laboratory of Mechanics in Energy Engineering, Shanghai, 200072, China \\ * donzhang@staff.shu.edu.cn
}

Introduction. Real-time monitoring of the operating state of rotating objects is important for safety assessments. Conventional DIC algorithms generally only consider translation and ignore the influence of rotation. It causes decorrelation if a large rotation occurs between the undeformed and deformed images ${ }^{(1)}$. Efforts ${ }^{(2)(3)}$ have been made on algorithms for fullfield measurements considering rotation. However, a real-time DIC algorithm for rotation measurement is of great interest for engineering purposes. In this work, an efficient DIC method has been proposed to achieve real-time rotational matching.

Methodology. A typical DIC algorithm mainly consisting of two consecutive processes: integer pixel searching and subpixel registration. In integer pixel searching stage, a new integer pixel search scheme based on contrarotation of the reference subset has been proposed. In this algorithm, the reference subset is reconstructed with the bicubic B-Spline interpolaton by rotating according to the rotation angle calculated from the deformed image in a reverse direction (as shown in Fig.1). Then the search is carried out in the deformed image by collecting the greyscale arrays in each regular subset in integer pixel domain.

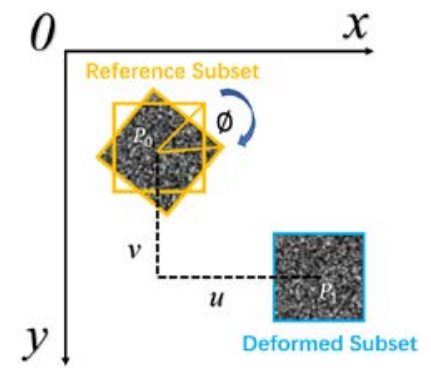

Fig.1 Image matching strategy considering rotation

A revised particle swarm optimization (PSO) algorithm has been developed based on a seed optimization scheme to improve the searching speed. As the sequential images are continuously recorded, the relative displacement between the adjacent images is small. Therefore, particles can be placed around the target in the previous frame. As shown in Fig.2, the target, which is obtained from the previous displacement calculation, is centered in the search area. Nine particles are initially placed around the target, and the rest of particles are randomly distributed in the search area. A threshold is predefined to terminate the iteration if the maximum correlation coefficient reaches a certain magnitude. Combined with block-based gradient descent search (BBGDS) algorithm, it is possible to locate the target within a limited time.

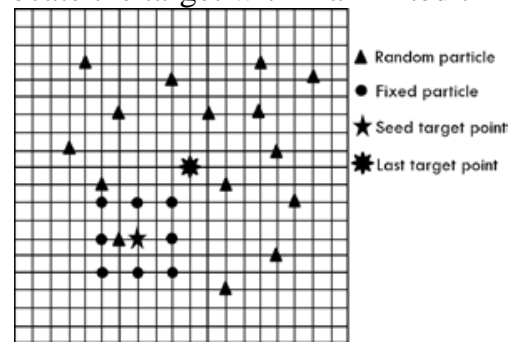

Fig.2 Arrangement of initial particles in PSO

The resulted target is used as the initial guess in the following sub-pixel registration. An improved forward additive Newton-Raphson (FA-NR) algorithm has been proposed to determine the location with sub-pixel accuracy. A zero-order shape function is adopted. The rotation angle is treated as a new variable as well as displacement components $(u, v)$ for iteration and optimization. Real-time tracking has been achieved by introducing the parallel computing strategy based on multi-core CPU.

Discussion and conclusion. Three parameters including the convergence range, threshold in PSO, and the density of the particles, in the proposed DIC method have been analyzed. It is found that the convergence radius of the improved FA-NR method is less than 3 pixels. This requires the threshold of the corelation coefficient to be greater than or equal to 0.75 in integer domain. In order to achieve the best computing efficiency, the density of the particles in the searching area is expected as $2.5 \%-3.75 \%$. The accuracy of the proposed method is as the same level as the traditinal DIC approach, which is considered to be 0.005 pixels. This algorithm has been applied to characteration of the displacement components of a rotating fan. The experimental analysis shows that the proposed method possesses high computational efficiency, and a stable displacement output for 6 points at $15 \mathrm{~Hz}$ is achieved with the existing image acuisition system with a frame rate of $25 \mathrm{fps}$.

This work is supported by the National Key R\&D Program of China \#2018YFF01014200, National Natural Science Foundation of China through grants \#11672347, \#11727804 and \#51732008.

\section{References.}

(1) B. Pan, K.M. Qian, H. Xie, A. Asundi. Meas Sci Technol, 20 (2009), pp. 062001-062018

(2) X. Zhang, J. Chen, Z. Wang, N. Zhan, R. Wang. Opt Lasers Eng, 50 (2012), pp. 922-926

(3) R. Wu, H. Qian, D. Zhang. Meas Sci Technol, 27 (2016), pp. 105002-105009 